\title{
A Fuzzy Expert Model of Haptic Perception for Automobile Touch-Screen Displays
}

\author{
Liviu-Cristian Duţu, ${ }^{1}$ Gilles Mauris, ${ }^{1}$ Philippe Bolon, ${ }^{1}$ Jean-Marc Tissot ${ }^{2}$ \\ ${ }^{1}$ Univ. Savoie Mont Blanc, LISTIC, F-74000 Annecy, France \\ ${ }^{1}$ \{liviu-cristian.dutu, gilles.mauris, philippe.bolon\}@univ-savoie.fr \\ ${ }^{2}$ Valeo Interior Controls, Annemasse, France \\ 2jean-marc.tissot@valeo.com
}

\begin{abstract}
Haptic feedback is currently emerging as a feasible solution to cope with the security-related issues of automobile touch-screen displays, and at the same time to improve users satisfaction and quality of use. Therefore, we have developed a fuzzy symbolic model of haptic perception for automobile interfaces in cooperation with an automotive ergonomics expert. The model predicts the induced comfort degree of the haptic effects based on their ergonomic properties, and achieves, on a set of 48 haptic patterns, a global error rate of $14.6 \%$ and a compatibility rate of $89.6 \%$ with the expert evaluations.
\end{abstract}

Keywords: Haptic interfaces, expert knowledge, sensory evaluations, linguistic modeling, automotive ergonomics

\section{Introduction}

During the last years, the electronic equipment market was dominated by the technological developments achieved in the field of touch-screen devices. Based on a perpetual growth, the number of touchsensitive equipments has already surpassed the psychological threshold of 1 billion units [1].

While smartphones and tablets still represent the main components of this growth, the tactile technology has recently expanded to new markets and products. Therefore, a large range of consumer products have already adopted the touch-screen technology, with some notable examples including: information panels, ATMs, digital cameras, remote controllers or hand watches.

To cope with the increasing customers demands, the automotive industry made significant progresses in this direction, and nowadays touch-sensitive screens have already replaced part of the mechanical interfaces in many modern automobiles [2].

Nevertheless, the conservative nature of the automotive industry pushed manufacturers to study the effects of this transition on passengers safety in a driving situation. These concerns are justified by the well-known fact that driver's engagement with in-car systems can interfere with the main driving task, negatively affecting it [3].
While still obeying to this general condition, current automobile touch-screen interfaces present an additional drawback, which is the lack of mechanical feedback from the device. Indeed, current touchscreen interfaces are passive, because unlike mechanical buttons, the virtual buttons on such displays do not actively stimulate the user's tactile sense. In a driving situation, this mechanical passivity adds additional pressure on the user's visual resources, detracting attention from the main driving task, with negative effects on safety [4]. This is corroborated by traffic security studies [5] showing that taking the eyes off the road for a duration longer than 2 seconds can significantly increase the risk of car accidents.

Faced with such clear evidence, car manufacturers latest efforts aimed at making the interaction with touch-screen displays safer for the end-user. As some researchers point out $[6,7]$, a possible way to achieve this is through an additional vibratory feedback, which is to be delivered by the tactile surface directly to the user finger on screen interaction.

Indeed, short vibrational stimuli, delivered when pushing a virtual touch-screen button, carry an important acknowledgment information about the state of the ongoing interaction between the user and the interface. As a consequence of this active stimulation of the tactile sense, user's visual resources are partially relieved. Moreover, since feedback information is now delivered through multiple senses simultaneously (vision and touch), the global cognitive workload can be reduced, according to the multiple resources theory [8].

However, while answering most of the aforementioned safety issues, the use of vibrotactile patterns opens a new and relatively unexplored dimension in automobile ergonomic sciences. Stimuli perception, adaptability and, ultimately, induced comfort are all important aspects that need to be tackled by the automotive industry in the near future.

In this regard, the present paper introduces a novel model of vibrotactile perception based on the evaluations of an expert in automotive ergonomics. Using fuzzy logic and privileged expert knowledge, the proposed model explores the connections between the induced comfort of vibrotactile signals 
and their evoked sensory parameters, in the context of in-vehicle information systems. Therefore, an ergonomic vision of haptic perception is introduced, with implications in the design and customization of automobile vibratory patterns.

The rest of this paper is organized as follows. In Section 2 we will present a literature overview on the subject of haptic feedback, with a special consideration for those studies dealing with comfort-related issues or automotive ergonomics. Next, in Section 3, the experiments carried out with the aid of an expert are detailed, and the knowledge acquired by questioning the expert is presented. Then, in Section 4, the fuzzy symbolic model of automobile haptic perception is introduced and validated based on the expert knowledge acquired above. Finally, in Section 5, some concluding remarks are drawn.

\section{Ergonomic Overview on Haptic Feedback}

From a psychophysical point of view, the perceptual aspects of vibrotactile stimulation became an important research topic toward the end of the 1960s. During that time, many important contributions, such as the four channels theory [9] and the Stevens power law of tactile vibration [10], were introduced.

However, it was only at the beginning of the 2000s that the first major studies dealing with the ergonomic aspects of haptic feedback appeared. This period coincides with the development of the first touch-screen consumer devices and also with the first attempts to enhance their usability via vibrotactile stimulation.

In a pioneering work [11], the authors show that the time spent scrolling a list on a PDA can be reduced by up to $22 \%$ when haptic feedback is present. Subsequent investigations, carried out by Brewster [12], show that the accuracy of phrase entering through a virtual keyboard on a touch-screen tablet can be improved by the haptic modality. This resulted both in a lower number of errors and a higher number of text lines entered. In a similar study, Hoggan et al. [13] revealed that, when haptic feedback is active, phrase entering on a touchscreen mobile phone was also faster, as compared to the non-haptic modality. Moreover, in the same study, the authors show that haptic feedback has a positive influence on the users mental workload, frustration and subjective performance level.

Therefore, it seems thus that vibrotactile stimulation, i.e. haptic feedback, improves both the usability and the cognitive workload associated with touch-screen interfaces.

Following the important success reported by the use of haptic feedback in general consumer devices, recently, the automotive industry began exploring this modality for their tactile in-vehicle information systems, as a way to improve the safety standards in a driving condition.

Therefore, in [6], the interaction with a tactile in-vehicle information system is studied for two distinct situations: a) using visual feedback only, and b) using bimodal visual-haptic feedback. The experiments were carried out in a driving simulator and required participants to perform a given task on the tactile surface, while engaged in the main driving task. The study reveled that in the second situation, i.e. using a joint visual-haptic feedback, both the duration of an eye glance and the number of eye glances required for a given task are reduced. This resulted in a global reduction of the task completion time, allowing the driver to focus more visual resources on the main driving task.

The above findings are corroborated by different studies on the same topic, which provide further evidence on the benefits of vibrotactile stimulation for automobile touch-screen displays. Therefore, in [14] the authors found that enabling haptic feedback for touch-screen interaction, while engaged in a virtual driving task, reduced the number of mistakes made, as compared to the non-haptic condition. Moreover, in [15], the effect of multi-modal feedback on drivers workload was estimated through a questionnaire. On this subject, the authors showed that the visual-haptic condition helped reduce the average workload score by more than $11 \%$ compared to the visual condition alone.

While these studies all assess the benefits of haptic feedback for automobile touch-screen displays, they do not provide explicit information on the $h e$ donic aspects of vibrotactile stimulation. Questions like: "which ergonomic variables most influence invehicle vibrotactile perception?" or "how to design, based on these variables, a set of pleasant and comfortable haptic stimuli?" still need to be answered.

In our previous studies [16] the comfort induced by vibrotactile stimuli was investigated from a $m e$ chanical perspective, using a set of measured variables associated with human skin's mechanoreceptors. The fuzzy model thus obtained proved useful in determining how the different activation degrees of the mechanoreceptors (as a result of the vibrotactile stimulation) influence the perceived comfort of the haptic stimuli.

However, the model in [16] decomposes the notion of vibrotactile comfort onto a set of technical variables, e.g. the stimulus energy within a certain frequency bandwidth or the positive velocity of the vibration. These variables are hard to understand by non-technical users and do not clearly reflect ergonomic expert knowledge in the field. This fact limits the explanatory power of the model.

In order to overcome this major drawback, it has been suggested that the comfort degree induced by vibrotactile stimuli should be decomposed onto a set of sensory parameters, closely related with the subjective aspects of haptic perception. Therefore, a complete experimental procedure was established with the aim of relating the sensory features of the vibrational signals with the comfort degree they in- 
duce. The proposed experimental procedure, along with the vibrotactile signals employed and the apparatus used to generate them, are presented in the following section.

\section{Experimental Procedure and Apparatus}

The experimental procedure was designed with the aid of an expert in automotive ergonomics having an experience of more than ten years in the field. It took place at the Interior Controls Research Laboratory, in the headquarters of the automotive manufacturer Valeo, in Annemasse, France.

\subsection{Apparatus and Stimuli}

In order to generate a wide range of vibrotactile signals, a novel and dedicated experimental device was used in this study. It was developed by the consortium of the MISAC project (Multi-fonctional Intelligence Surface for Automotive $\&$ Aeronautics Cockpits) with the intent to boost research on vibrotactile stimulation for future touch-screen interfaces installed in cars and airplanes cockpits.

The apparatus consists of a capacitive touchscreen device able to deliver haptic feedback through an array of piezoelectric actuators disposed under the screen layer. Thanks to the original architecture of the actuators, the vibrotactile signals generated cover the frequency range of 20-300 Hz, and can reach acceleration amplitudes of up to $40 \mathrm{G}$ peak-to-peak. The experimental device is connected to a $\mathrm{PC}$ which sends to the actuators the appropriate parameters of the haptic effects to be triggered, and which also stores the subjective evaluations of the expert. A complete illustration of the apparatus is shown in Figure 1. The virtual interface of the tactile screen is displayed in Figure 2.

The above experimental device allowed the generation of 48 unique haptic effects, spanning 12 frequencies between $30 \mathrm{~Hz}$ and $300 \mathrm{~Hz}$, two durations, i.e. short and long, and two waveforms, i.e. triangle and sine. Given the wide covering of the input parameters, the set of 48 haptic stimuli was considered complete and representative for an automobile haptic touch-screen interface.

\subsection{Experimental Procedure}

To let the expert assess both the sensory parameters of the haptic effects and their induced comfort degree, the experimental procedure was divided into two different stages, corresponding to the two analyses the expert had to perform: a sensory evaluation for the parameters of the vibrotactile stimuli followed by a hedonic evaluation.

\subsubsection{Sensory analysis of the haptic effects}

According to his experience in automotive ergonomics, the expert identified the following sensory parameters of the haptic effects as being the

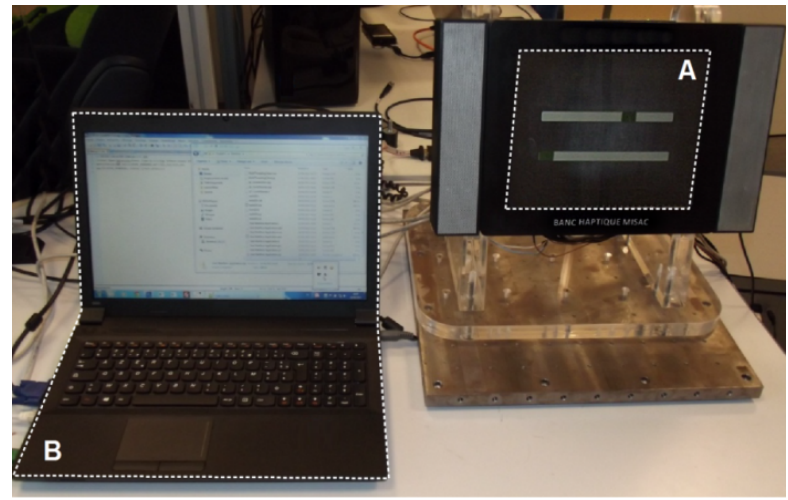

Figure 1: The experimental device used: A) Capacitive touch-screen. The actuators are disposed under the screen. B) The PC communication system.

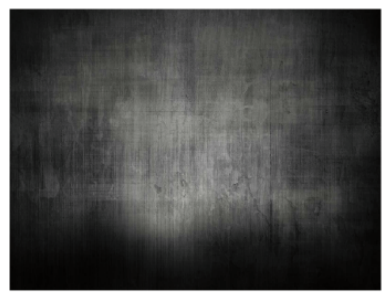

(a)

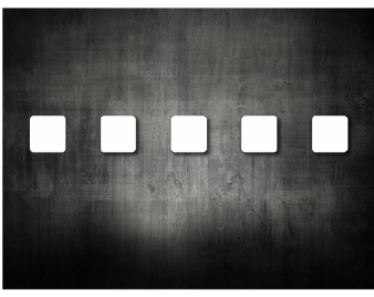

(b)
Figure 2: Touch-screen virtual interface presenting: (a) the background; (b) a series of five virtual buttons, each associated with an unique haptic effect triggered when the button is pushed.

most relevant ones for automobile touch-screen interfaces:

- Adaptability of the haptic effect to the automobile driving context: property assessing whether the haptic effect is well-adapted to be used in automobile touch-screen interfaces; "context adaptability" assimilates several ergonomic characteristics of the haptic effects, such as intensity, stimulus responsiveness or perceptual duration.

- Neatness: ergonomic criterion assessing the "perceptual cleanness" of the haptic effect; a neat vibrotactile stimulus should be sharp and have no parasite vibrations; otherwise the stimulus would be considered noisy.

- Sensation of displacement: sensory property used to assess the perceptual sensation that the finger "sinks" into the tactile surface as a result of the vibrotactile stimulus received; the "sinking sensation" induced by the haptic effect can therefore create the impression of pushing a mechanical button;

Furthermore, for each of the above properties, the expert proposed several classes, as linguistic labels, which can be consulted in Table 1. Therefore, the three properties chosen denote the most salient ergonomic features of the vibrotactile signals. The granularity of each property reflects the expert's dis- 
crimination ability, i.e. how many classes of haptic effects can he discriminate for the given criterion.

Therefore, using the apparatus described in Section 3.1, the expert evaluated (by touching the capacitive screen with the fingertip) each of the 48 haptic effects generated, according to the above properties. Hence, for each sensory property, each haptic effect was assigned to one of their corresponding classes. The 48 haptic effects were randomly presented, and the experience was repeated two times. After the two independent runs, the final linguistic label, i.e. class, associated with the haptic effect was considered to be the aggregation of the classes given by the expert in the two runs. Table 2 shows the complete list of possible aggregations for the Adaptability parameter. Please note that the "+" operator represents fuzzy sets union, rather that numerical summation [17]. This form of representation allows expert hesitancy to be translated into term graduality, as opposed to forcing an exclusive term. The same methodology was employed for the two other parameters, i.e. Neatness and Displacement.

Therefore, after the two evaluations we obtain a fuzzy linguistic description for the given haptic effect. Please note that in the rest of the paper, whenever a linguistic label's membership degree is 0 , it will be omitted from the description.

The fuzzy linguistic descriptions evaluated by the expert for the three sensory properties, and for each haptic effect, will be later used as input variables for the fuzzy symbolic model of automobile vibrotactile perception.

\subsubsection{Hedonic analysis of the haptic effects}

The second stage of the experimental procedure consisted in allowing the expert to evaluate the induced comfort degree for each haptic effect. Using the same experimental device, the expert evaluated each haptic effect (once again by touching the capacitive screen with the fingertip) and rated the perceived comfort degree induced by the effect. The rates were given on a scale with 5 labels: $\{$ "Very $U n$ comfortable", "Uncomfortable", "Neutral", "Comfortable" and "Very Comfortable" . Therefore, each label can be seen as a fuzzy description of the comfort degree.

Moreover, during the experiment, the expert placed himself in a virtual driving situation, and evaluated the contextual comfort degree induced by the stimuli. More precisely, according to his expertise, he evaluated the pleasantness of the touchscreen haptic effects, as they would be perceived inside a running automobile.

This procedure was repeated three times, allowing to obtain three evaluations on the perceived comfort degree for each haptic effect. The global comfort evaluation was obtained by the same aggregation methodology detailed in Table 2 for the

\begin{tabular}{|l|c|}
\hline Criterion & Classes \\
\hline \multirow{4}{*}{ Adaptability } & Good \\
\cline { 2 - 2 } & Acceptable \\
\cline { 2 - 2 } Neatness & Unacceptable \\
\hline \hline \multirow{4}{*}{ Sensation of Displacement } & Neat \\
\cline { 2 - 2 } & Average \\
\cline { 2 - 2 } & Noisy \\
\cline { 2 - 2 } & Average \\
\cline { 2 - 2 } & Too Much \\
\cline { 2 - 2 } & Low \\
\cline { 2 - 2 } & None \\
\hline
\end{tabular}

Table 1: Linguistic labels for the sensory criteria defined by the expert.

Adaptability parameter. For instance, if the evaluations given in the three runs of the experiment for haptic effect $i$, are Comfortable, Neutral and Neutral, respectively, the final comfort evaluation is:

$$
\begin{aligned}
\operatorname{Comfort}(i)= & 0.33 / \text { Comfortable }+ \\
& 0.66 / \text { Neutral } .
\end{aligned}
$$

\subsection{Expert Knowledge Rulebase of Vibrotactile Perception}

Therefore, each haptic effect is now characterized by the evaluations on the sensory parameters, on the one hand, and by the evaluation on the comfort degree, on the other hand. In order to model expert's knowledge on automobile vibrotactile perception, these two separate knowledge layers must be connected.

Given the fact that the evaluations obtained on the sensory parameters are expressed as fuzzy linguistic descriptions without having a numerical universe of discourse associated, in this study we have chosen a fuzzy symbolic model [18] to link these evaluations to the ones on the induced comfort degree. We recall that a fuzzy symbolic model uses linguistic labels for the input and output variables, but unlike Mamdani systems, the output labels are treated as symbols, and not as actual fuzzy subsets.

Therefore, the input variables of the proposed model are the evaluations on the sensory parameters, and the output variable is represented by the evaluations on the comfort degree induced by the haptic effects. The system rule-base was defined together with the expert, and consists of 45 $(3 \times 3 \times 5)$ fuzzy "IF-THEN" statements relating the above variables. Due to space limitations, Table 3 presents a collection of only 15 among the 45 rules used.

Please notice that for certain combinations of the antecedents, the system's output, i.e. the perceived 


\begin{tabular}{|c|c|c|}
\hline First Evaluation & Second Evaluation & Final Linguistic Label \\
\hline \hline Good & Good & $1 /$ Good $+0 /$ Acceptable + 0/Unacceptable \\
\hline Good & Acceptable & $0.5 /$ Good $+0.5 /$ Acceptable + 0/Unacceptable \\
\hline Good & Unacceptable & $0 /$ Good $+1 /$ Acceptable $+0 /$ Unacceptable \\
\hline \hline Acceptable & Good & $0.5 /$ Good $+0.5 /$ Acceptable $+0 /$ Unacceptable \\
\hline Acceptable & Acceptable & $0 /$ Good $+1 /$ Acceptable $+0 /$ Unacceptable \\
\hline Acceptable & Unacceptable & $0 /$ Good $+0.5 /$ Acceptable $+0.5 /$ Unacceptable \\
\hline \hline Unacceptable & Good & $0 /$ Good $+1 /$ Acceptable $+0 /$ Unacceptable \\
\hline Unacceptable & Acceptable & $0 /$ Good 0.5/Acceptable $+0.5 /$ Unacceptable \\
\hline Unacceptable & Unacceptable & $0 /$ Good $+0 /$ Acceptable $+1 /$ Unacceptable \\
\hline
\end{tabular}

Table 2: List of possible aggregations for the Adaptability parameter.

\begin{tabular}{|c|c|c|c|c|}
\hline & Adaptability & Neatness & Displacement & Perceived Comfort \\
\hline \hline$R_{1}$ & Good & Neat & Adapted & $1 /$ Very Comfortable \\
\hline$R_{2}$ & Good & Neat & Low & $0.5 /$ Neutral $+0.5 /$ Comfortable \\
\hline$R_{3}$ & Good & Average & Adapted & $1 /$ Comfortable \\
\hline$R_{4}$ & Good & Average & Low & $1 /$ Neutral \\
\hline$R_{5}$ & Good & Noisy & Low & $0.5 /$ Uncomfortable $+0.5 /$ Neutral \\
\hline \hline$R_{6}$ & Acceptable & Neat & Adapted & $0.5 /$ Comfortable $+0.5 /$ Very Comfortable \\
\hline$R_{7}$ & Acceptable & Neat & Low & $1 /$ Neutral \\
\hline$R_{8}$ & Acceptable & Average & Average & $1 /$ Neutral \\
\hline$R_{9}$ & Acceptable & Average & Low & $0.5 /$ Uncomfortable $+0.5 /$ Neutral \\
\hline$R_{10}$ & Acceptable & Noisy & None & $1 /$ Uncomfortable \\
\hline \hline$R_{11}$ & Unacceptable & Neat & Too Much & $1 /$ Neutral \\
\hline$R_{12}$ & Unacceptable & Neat & Low & $1 /$ Uncomfortable \\
\hline$R_{13}$ & Unacceptable & Average & Adapted & $1 /$ Very Uncomfortable \\
\hline$R_{14}$ & Unacceptable & Average & None & $1 /$ Very Uncomfortable \\
\hline$R_{15}$ & Unacceptable & Noisy & Low & \\
\hline
\end{tabular}

Table 3: Expert Knowledge Rulebase of Automobile Vibrotactile Perception.

comfort degree, is given as a fuzzy linguistic description, rather than by a single label, as is the case in $R_{5}$ for example. This fact is related to the expert's hesitation on the label to be associated with the given antecedents. However, a sum of the membership degrees equal to 1 was imposed in those cases.

Since the defined rule-base is to be used for further developments of haptic feedback touch-screen interfaces, its consistency must be first validated. For this purpose, in Section 4 the fuzzy symbolic model of vibrotactile perception is detailed, and its quantitative aspects are used to assess the adequacy of the rule-base.

\section{Fuzzy Symbolic Model of Vibrotactile Perception}

The expert's evaluations on the sensory parameters and those on the perceived comfort degree, along with the fuzzy rules defined, represent the knowledge base of the fuzzy symbolic model. The inference between the input space of the sensory properties, to the output space of the perceived comfort degree, is accomplished by the projectioncombination principle [19].

Since a symbolic model does not automatically guarantee that the sum of the output degrees equals one, special care must be taken in choosing the inference operators, in order to avoid an inferred output of the type "0.6/Comfortable + $0.4 /$ Neutral $+0.7 /$ Uncomfortable". Although feasible, these representations are currently considered counter-intuitive by the users. Therefore, in [20] it is shown that a sum of the output degrees equal to 1 can be assured by the arithmetic product T-norm as conjunction operator, and the bounded sum $T$ conorm as projection operator:

$$
\begin{gathered}
\top(a, b)=a \times b ; \\
\perp(a, b)=\min (a+b, 1)
\end{gathered}
$$

Now, let $S$ be a haptic signal with the following 
fuzzy linguistic descriptions:

$$
\begin{aligned}
\text { Adaptability }(S) & =0.5 / \text { Good }+0.5 / \text { Acceptable } \\
\text { Neatness }(S) & =0.5 / \text { Neat }+0.5 / \text { Average } \\
\text { Displacement }(S) & =1 / \text { Low }
\end{aligned}
$$

Using the operators in (1), the rules activated by the above inputs are $R_{2}, R_{4}, R_{7}, R_{9}$. Each rule has an activation degree of 0.25 , which corresponds to the product of the haptic signal inputs. Next, the membership degree for each output symbol is calculated. Below we detail this reasoning for the output symbol Neutral.

The Neutral symbol can be found in all the activated rules, and in order to compute its global membership degree, we must first compute its partial membership degree in each rule. This gives:

$$
\begin{aligned}
& \text { Out } t_{\text {Neutral }}^{R_{2}}=0.125 / \text { Neutral } \\
& O u t_{\text {Neutral }}^{R_{4}}=0.25 / \text { Neutral } \\
& \text { Out } t_{\text {Neutral }}^{R_{7}}=0.25 / \text { Neutral } \\
& \text { Out } t_{\text {Neutral }}^{R_{9}}=0.125 / \text { Neutral }
\end{aligned}
$$

Using the bounded sum T-conorm, the final membership degree is:

$$
\text { Out }_{\text {Neutral }}=0.75 / \text { Neutral }
$$

Iterating this procedure for each symbol gives the inferred symbolic output of the system:

$$
\begin{aligned}
\text { Out }= & 0.125 / \text { Com fortable }+0.75 / \text { Neutral }+ \\
& 0.125 / \text { Uncomfortable }
\end{aligned}
$$

In order to validate the consistency of the rule base used, the inferred symbolic output of the system must be compared with the actual output, i.e. the comfort evaluations assessed by the expert. Although some measures based on symbolic distances between the two have been proposed in the literature for this purpose [21], we have decided to defuzzify both outputs and compare them.

Therefore, a set of modal or characteristic values $M_{i} \in \Re$ have been assigned to the five output labels in order to obtain a crisp numerical value for the inferred output. This is accomplished using the height method of defuzzification [22], as described below:

$$
y=\frac{\sum_{i=1}^{K_{\text {Out }}} \mu_{C_{i}} \cdot M_{i}}{\sum_{i=1}^{K_{\text {Out }}} \mu_{C_{i}}}
$$

where $K_{\text {Out }}=5$ is the number of output symbols and $\mu_{C_{i}}$ is the membership degree of the haptic effect to the output class $C_{i}$.

For the fuzzy symbolic model of vibrotactile perception, the modal values assigned to the output symbols \{ "Very Uncomfortable", "Uncomfortable", "Neutral", "Comfortable" and "Very Comfortable" are $\{-2,-1,0,+1,+2\}$, respectively. The set of modal values was chosen such that it reflects the bipolar nature of the linguistic scale used. Moreover, the equidistant repartition of the modal values indicates that the same perceptual discrepancy was considered between any two adjacent symbols, which seems in agreement with the expert's own knowledge on the matter.

\subsection{Experimental Results}

Using the evaluations provided by the expert for the set of 48 haptic effects, a quantitative analysis of the symbolic model was carried out. The performance indicators used are the Mean Square Error of the system $(M S E)$ and a normalized error measure $\Delta$, defined below:

$$
\begin{gathered}
\Delta=\frac{\sum_{i=1}^{M}|P(i)-A(i)|}{\sum_{i=1}^{M} \operatorname{MaxErr}_{i}}, \\
\text { with } \\
\operatorname{MaxErr}_{i}=\left\{\begin{array}{c}
A(i)-\min \left(I_{A}\right), \text { if } A(i) \geq k \\
\max \left(I_{A}\right)-A(i), \text { otherwise }
\end{array}\right.
\end{gathered}
$$

where $M$ is the number of haptic effects evaluated; $P$ is the array containing the predicted comfort values, $A$ is the array containing the actual comfort values evaluated by the expert; MaxErr ${ }_{i}$ is the largest error the system can make when forecasting the comfort value of the haptic effect $i ; I_{A}$ is the interval of variation of the comfort value, i.e. $I_{A}=[-2,2]$, and $k$ is its midpoint.

Furthermore, in order to easily detect the incompatibilities between the haptic effects and the fuzzy model, an evaluation tolerance of $U=0.8$, i.e. $20 \%$ of $I_{A}$, was used. This value is related to the human evaluation imprecision for vibrotactile stimuli, and was previously agreed with the expert. Therefore, any haptic effect with an absolute difference between its actual and predicted comfort degrees greater than $U=0.8$, will be considered incompatible with the model. Let $N_{I C}$ be the number of incompatible haptic effects. Then the compatibility rate $(C R)$ of the system is:

$$
C R=1-\frac{N_{I C}}{M}[\%]
$$

On the above performance indicators, the results of the fuzzy model for the set of 48 haptic effects are collected in Table 4. A quick analysis of the results reveals that $89.6 \%$ of the haptic effects are compatible with the perception model defined, and that the global error of the system is $14.6 \%$. A caseby-case comparison between the predicted and actual comfort values is shown in Figure 3, where the $N_{I C}=5$ incompatible effects are marked by thick vertical lines. Therefore, for most haptic effects, the model accurately predicts the comfort value, based on their evoked ergonomic properties.

Finally, let us point out that the system global error $(\Delta)$ and compatibility rate $(C R)$ are not affected by the actual choice of the modal values, as long as 


\begin{tabular}{|c|c|}
\hline Indicator & Result \\
\hline \hline Global Error $(\Delta)$ & $14.60 \%$ \\
\hline Compatibility Rate $(C R)$ & $89.6 \%\left(N_{I C}=5\right)$ \\
\hline Mean Square Error $(M S E)$ & 0.275 \\
\hline
\end{tabular}

Table 4: Results of the Fuzzy Symbolic Model for the Set of 48 Haptic Effects.

they are uniformly spaced. For instance, the same global error and compatibility rate are obtained using the set of modal values $\{-1,-0.5,0,+0.5,+1\}$.

\subsection{Discussion}

The good quantitative results obtained validate the adequacy of the rule-base used, which can be seen as an ergonomic knowledge base for in-vehicle vibrotactile perception.

A post-hoc analysis of the $N_{I C}=5$ incompatible haptic effects reveals they are not uniformly distributed, but follow a rather specific pattern. Therefore, three out of the five incompatible haptic effects have an Adaptability $=1 /$ Acceptable and one of them has an Adaptability $=0.5 /$ Acceptable + $0.5 /$ Unacceptable. This analysis shows that the model is less accurate in the intermediate sector of the Adaptability property, i.e. where the ergonomic adaptability of the haptic effects to the automobile context is in the region of Acceptable.

These findings indicate a potential hesitation of the expert for those haptic effects which are not clearly positioned on the Adaptability scale. They also suggest that Context Adaptability is the most important ergonomic property of the haptic effects, which is in agreement with the expert reasoning.

Nevertheless, the global behavior of the fuzzy model on the analyzed set of haptic effects is considered by the expert to be congruent with his own ergonomic vision on vibrotactile perception. Moreover, the model performance indicators are considered relevant, as for most of the effects, the system predictions match the subjective evaluations of the expert, within a reasonable interval.

\section{Conclusion}

In the present paper a fuzzy expert model of haptic perception for automobile touch-screen displays was proposed. The model presents an ergonomic vision on vibrotactile perception, and decomposes the notion of "haptic comfort" onto a series of sensory properties, which are specific to the automotive industry, e.g. Adaptability, Neatness, and Sensation of Displacement.

A set of 48 haptic effects, generated on a state-ofthe-art experimental device, were evaluated by an expert in automotive ergonomics and interior controls. These evaluations fully characterize the ergonomic features of the haptic effects, as well as their induced comfort degree.

Based on the above evaluations and using an interpretable linguistic rule-base, a fuzzy symbolic model was defined. The model achieves a global error rate of $14.6 \%$, and manages to correctly predict the comfort degree for $89.6 \%$ of the haptic effects studied, within a predefined precision threshold. Therefore, the model was found to be in agreement with the expert's ergonomic knowledge on the matter, and it could be used for more complex haptic effects, such as those associated with virtual knobs or sliders.

An interesting perspective would be to test the proposed model for touch-screen aeronautical instrument panels, given the similarities they bear with automobile tactile interfaces. In this regard, the evaluations of an expert in aeronautical cockpit design, member of the MISAC consortium, could be used to validate or adjust the proposed model.

\section{Acknowledgment}

This study was developed under the FUI-MISAC project ("Multi-functional Intelligent Surface for Automotive 83 Aeronautics Cockpits"), approved by the French Government with the contract number F-11-06-048-V.

\section{References}

[1] Y. Duke, Global Touch-Screen Panel Shipments to Double by 2016, IHS Analyst Announces at SID. Market report, IHS Inc., May 2013.

[2] H. Kim and H. Song. Evaluation of the safety and usability of touch gestures in operating invehicle information systems with visual occlusion. Applied ergonomics, 45(3):789-798, Elsevier, 2014.

[3] J. B. Van Erp and H. A. H. C. Van Veen. Vibrotactile information presentation in automobiles. In Proceedings of Eurohaptics, pages 99-104, 2001.

[4] A. Stevens, A. Quimby, A. Board, T. Kersloot and P. Burns. Design guidelines for safety of invehicle information systems. Technical Report, TRL Limited, 2002.

[5] S. G. Klauer, T. A. Dingus, V. L. Neale, J. D. Sudweeks and D. J. Ramsey. The impact of driver inattention on near-crash/crash risk: An analysis using the 100-car naturalistic driving study data. Report No. DOT HS 810 594, National Highway Traffic Safety Administration, Washington DC, 2006.

[6] M. J. Pitts, G. Burnett, L. Skrypchuk, T. Wellings, A. Attridge and M. A. Williams. Visual-haptic feedback interaction in automotive touchscreens. Displays, 33(1):7-16, 2012.

[7] S. Dabic, J. Navarro, J. M. Tissot and R. Versace. User perceptions and evaluations of short 


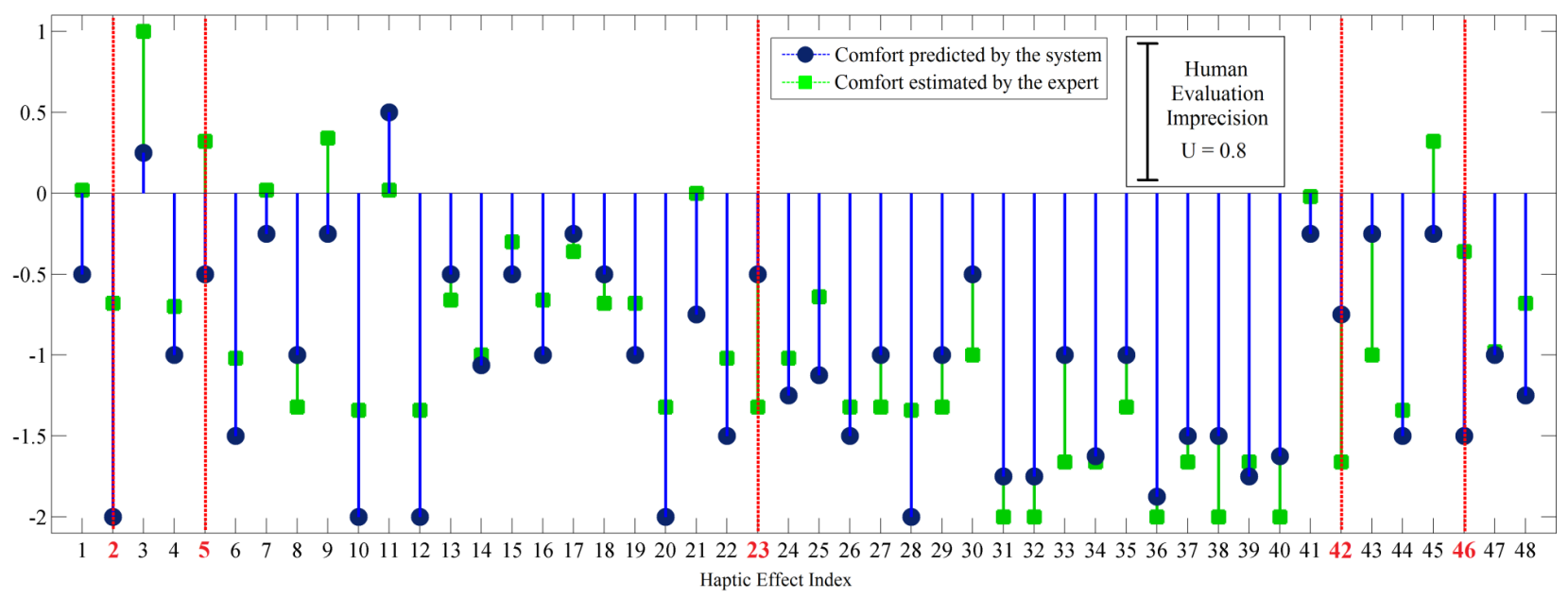

Figure 3: Comparison between the predicted and actual comfort degrees for the set of 48 haptic effects. In the above figure, the abscissa holds the indexes of the different haptic effects (in no particular order), while the ordinate axis represent the numerical scale of attribute comfort.

vibrotactile feedback. Journal of Cognitive Psychology, 25(3):299-308, 2013.

[8] C. D. Wickens. Multiple resources and performance prediction. Theoretical issues in ergonomics science, 3(2):159-177, 2002.

[9] S. J. Bolanowski, G. A. Gescheider, R. T. Verrillo and C. M. Checkosky. Four channels mediate the mechanical aspects of touch. The Journal of the Acoustical society of America, 84(5):16801694, 1988.

[10] S. S. Stevens. Tactile vibration: Change of exponent with frequency. Perception and Psychophysics, 3(3):223-228, 1968.

[11] I. Poupyrev, S. Maruyama and J. Rekimoto. Ambient touch: designing tactile interfaces for handheld devices. In Proceedings of the 15th Annual ACM Symposium on User Interface Software and Technology, pages 51-60. ACM, 2002.

[12] S. Brewster, F. Chohan and L. Brown. Tactile feedback for mobile interactions. In Proceedings of the SIGCHI Conference on Human Factors in Computing Systems, pages 159-162. ACM, 2007.

[13] E. Hoggan, S. Brewster J. Johnston. Investigating the effectiveness of tactile feedback for mobile touchscreens. In Proceedings of the SIGCHI Conference on Human Factors in Computing Systems, pages 1573-1582. ACM, 2008.

[14] H. Richter, R. Ecker, C. Deisler and A. Butz. HapTouch and the $2+1$ state model: potentials of haptic feedback on touch based in-vehicle information systems. In Proceedings of the 2nd International Conference on Automotive User Interfaces and Interactive Vehicular Applications, pages 72-79. ACM, 2010.

[15] J. H. Lee and C. Spence. Assessing the benefits of multimodal feedback on dual-task performance under demanding conditions. In Proceedings of the 22nd British HCI Group Annual Conference on People and Computers: Culture, Creativity, Interaction-Volume 1, pages 185
192. British Computer Society, 2008.

[16] L. C. Duţu, G. Mauris, P. Bolon, S. Dabic and J. M. Tissot. A Fuzzy Rule-Based Haptic Perception Model for Automotive Vibrotactile Display. In Information Processing and Management of Uncertainty in Knowledge-Based Systems, pages 576-585. Springer International Publishing, 2014.

[17] L. A. Zadeh. Outline of a new approach to the analysis of complex systems and decision processes. IEEE Transactions on Systems, Man and Cybernetics, 1:28-44, 1973.

[18] G. Mauris, E. Benoit and L. Foulloy. Fuzzy symbolic sensors - From concept to applications. Measurement, 12(4):357-384, 1994.

[19] H. T. Nguyen, M. Sugeno, R. M. Tong and R. R. Yager. Theoretical aspects of fuzzy control, page 74, John Wiley and Sons, 1995.

[20] G. Mauris, E. Benoit and L. Foulloy. The aggregation of complementary information via fuzzy sensors. Measurement, 17(4):235-249, 1996.

[21] L. Foulloy and E. Benoit. Building a class of fuzzy equivalence relations. Fuzzy Sets and Systems, 157(11):1417-1437, 2006.

[22] B. Bouchon-Meunier, R. R. Yager, L. A. Zadeh (Eds.). Fuzzy Logic and Soft Computing. In World Scientific, 1995. 